\title{
Ibuprofen erhöht den Blutdruck
}

Das frei verkäufliche nicht steroidale Antirheumatikum Ibuprofen scheint weniger sicher zu sein als bisher gedacht. Im Vergleich zu anderen NSAR führt es zu einer relevanten Blutdruckerhöhung.

D as erhöhte kardiovaskuläre Risiko einiger nicht steroidaler Antirheumatika könnte zum Teil auf einem blutdrucksteigernden Effekt beruhen", berichtete Prof. Frank Ruschitzka von der Kardiologischen Universitätsklinik in Zürich. In der Doppelblindstudie waren 444 Patienten mit Arthrose oder rheumatoider Arthritis, bei denen kardiovaskuläre Risikofaktoren bestanden, unter Esomeprazol-Schutz in drei Armen mit Celecoxib (mittlere Dosis: 208 mg/Tag), Ibuprofen $(2.030 \mathrm{mg} / \mathrm{Tag})$ oder Naproxen $(852 \mathrm{mg} / \mathrm{Tag})$ behandelt worden. Primärer Endpunkt war die Veränderung des 24-Stunden-Blutdruckes nach viermonatiger Therapie.

\section{4-Stunden-Blutdruck steigt um $4 \mathrm{mmHg}$}

Celecoxib hatte nahezu keine Wirkung auf den systolischen und diastolischen 24-Stunden-Blutdruck $(-0,3 /+0,1 \mathrm{mmHg})$. Auch bei Naproxen fielen die Veränderungen moderat aus $(+1,6 /+0,7 \mathrm{mmHg})$. Bei Ibuprofen waren die Blutdruckveränderungen hingegen durchaus relevant $(+3,9 /+0,8 \mathrm{mmHg}) ; 23,2 \%$ der Patienten entwickelten eine Hypertonie, im Vergleich zu 19\% unter Naproxen und $10,3 \%$ unter Celecoxib.

„Die Ergebnisse sprechen einmal mehr dafür, dass Patienten mit kardiovaskulären Risikofaktoren den Arzt konsultieren sollten, bevor sie Schmerzmittel ein- nehmen“, äußerte sich Ruschitzka dazu auf dem ESC-Kongress. Auf Schmerzmittel zu verzichten, sei aber auch keine Lösung, weil unbehandelte Schmerzen ebenfalls einen kardiovaskulär relevanten Stressfaktor darstellen. Durch die richtige Wahl kann das Risiko aber minimiert werden.

Die PRECISION-ABPM-Studie war eine Substudie der Ende 2016 publizierten viel größeren PRECISION-Studie, die bei über 24.000 Patienten das kardiovaskuläre Nebenwirkungsrisiko der drei Antirheumatika verglichen hatte. Die Studie hatte gezeigt, dass Celecoxib den beiden Vergleichspräparaten nicht unterlegen war. In der On-treatment-Analyse hatte Celecoxib gegenüber Ibuprofen weniger kardiovaskuläre, weniger gastrointestinale und weniger renale Nebenwirkungen verursacht.

Dr. med. Dirk Einecke

\section{Kardioversion: NOAK reduziert Schlaganfall-Rate auf $0 \%$}

Apixaban schützt effektiver vor Schlaganfällen als eine konventionelle Therapie mit Heparin und einem Vitamin-K-Antagonisten, wenn Patienten mit Vorhofflimmern einer Kardioversion unterzogen werden - so das Ergebnis der EMANATE-Studie.

$\mathrm{D}$ en Sinusrhythmus via Kardioversion wiederherzustellen, ist ein integraler Therapiebaustein bei neu aufgetretenem symptomatischen Vorhofflimmern. Der Eingriff erfolgt in der Regel unter Antikoagulation mit Heparin und einem Vitamin-K-Antagonisten (VKA), um Schlaganfälle und systemische Embolien zu verhindern.

Post-hoc-Analysen der großen Studien RE-LY, ROCKET-AF, ARISTOTLE und ENGAGE-AF mit den neuen oralen Antikoagulanzien deuten darauf hin, dass eine Kardioversion unter diesen Substanzen mit ebenso niedrigen Komplikationsraten einher geht wie unter dem VKA Warfarin.

Für Rivaroxaban und Edoxaban liegen mit der X-VeRT-Studie und der ENSURE-AF-Studie inzwischen prospekti- ve Studien vor, welche die Substanzen eigens im Rahmen einer Kardioversion untersuchten und bei insgesamt niedrigen Komplikationsraten eine vergleichbare Wirksamkeit und Sicherheit im Vergleich zum konventionellen Vorgehen dokumentieren.

\subsection{Patienten mit kurzfristig geplanter Kardioversion}

Nun wurden Ergebnisse einer Studie mit dem Faktor-Xa-Hemmer Apixaban vorgestellt. In der EMANATE-Studie waren 1.500 zuvor nicht antikoagulierte Patienten mit Vorhofflimmern, die für eine rasche Kardioversion innerhalb von 48 Stunden anstanden, zwei Therapiearmen per Randomisierung zugeteilt worden. Die eine Gruppe wurde konventionell mit Heparin und Warfarin antiko- aguliert, die andere erhielt Apixaban $(2 \times$ $5 \mathrm{mg} / \mathrm{Tag}$ ), wobei zwei Stunden vor dem Eingriff eine zusätzliche Loading-Dose (10 mg) erlaubt war. Davon wurde bei etwas weniger als der Hälfte der Patienten Gebrauch gemacht. Bei bestimmten Risikokonstellationen wurden die Apixaban-Dosierungen jeweils halbiert.

Wie Studienautor Prof. Michael Ezekowitz von der Thomas Jefferson Universität in Philadelphia beim ESC-Kongress berichtete, erlitten sechs Patienten der Kontrollgruppe, aber kein Patient in der Apixaban-Gruppe einen Schlaganfall. Bei drei Patienten unter Apixaban sowie sechs Patienten unter Heparin/ Warfarin trat eine schwere Blutung auf, zwei Patienten unter Apixaban und ein Patient unter Heparin/Warfarin starben. Insgesamt waren die Komplikationsraten niedrig. Erkenntnisse über den Stellenwert der Loading-Dose lassen sich aus den wenigen Komplikationen nicht herauslesen.

„Wir sind überzeugt, dass die Ergebnisse der Studie dafür sprechen, den Faktor-Xa-Hemmer im Rahmen einer Kardioversion einzusetzen“, resümierte Ezekowitz.

Dr. med. Dirk Einecke 